\title{
Minimum-Latency Schedulings for Group Communications in Multi-channel Multihop Wireless Networks ${ }^{\star}$
}

\author{
Peng-Jun Wan ${ }^{1}$, Zhu Wang ${ }^{1}$, Zhiyuan Wan ${ }^{2}$, Scott C.-H. Huang ${ }^{2}$, and Hai Liu ${ }^{3}$ \\ 1 Illinois Institute of Technology, Chicago IL 60616, USA \\ 2 City Univesrsity of Hong Kong, Kowloon, Hong Kong \\ 3 Hong Kong Baptist University, Kowloon, Hong Kong
}

\begin{abstract}
This paper is motivated by exploring the impact of the number of channels on the achievable communication latency for a specific communication task. We focus on how to utilize the multiple channels to speed up four group communications including broadcast, aggregation, gathering, and gossiping in wireless networks under protocol interference model. Four scheduling algorithms are developed for these four group communications. We derive explicit tight bounds on the latencies of the four communication schedules produced by these algorithms. These latency bounds in general decrease with the number of channels and are also within constant factors of the respective minimum.
\end{abstract}

\section{Introduction}

With the rapid technology advances, many off-the-shelf wireless transceivers (i.e., radios) are capable of operating on multiple channels. For example, the IEEE $802.11 \mathrm{~b} / \mathrm{g}$ standard and IEEE 802.11a standard provide 3 and 12 channels respectively, and MICA2 sensor motes support more than 50 channels. This paper aims to conduct comprehensive algorithmic studies of minimizing the communication latency by utilizing multiple channels under the following network model. All network nodes $V$ lies in plane. Every node has a communication radius normalized to one, and an interference radius $\rho$ for some parameter $\rho \geq 1$. The communication (respectively, interference) range of a node $v \in V$ is the disk centered at $v$ of one (respectively, $\rho$ ). A node $v$ can receive the message successfully from a transmitting node $u$ if $v$ is within the transmission range of $u$ but is outside the interference range of any other node transmitting simultaneously at the same channel. Let $\lambda$ be the number of available channels. Then, each network is associated with a pair of parameters $(\lambda, \rho)$. We further assume that all communications proceeds in synchronous time-slots and each node can either transmit or receive at most one packet of a fixed size in each time-slot.

\footnotetext{
* This work was supported in part by NSF under grant CNS-0831831, and by the Research Grant Council of Hong Kong under the project CERG CityU 113807.
} 
In this paper, we focus on the communication schedules for the following four group communication tasks in multihop wireless networks: broadcast, data aggregation, data gathering, and gossiping. The problem of computing a broadcast (respectively, aggregation, gathering, gossiping) schedule with minimum latency is referred to Minimum-Latency Broadcast (respectively, Aggregation, Gathering, Gossiping) Schedule. There are a number of recent works on the scheduling for broadcasting 7811, aggregation 312 14, gathering 126] and gossiping [7810] subject to the protocol interference constraint or the $k$-hop interference model. All of them assume $\lambda=1$ (i.e. single-channel) and most of them assume $\rho=1$ (i.e. the interference radius equal to the communication radius). Consequently, the impact of multiple channels on communication latency in the more general and practical case with $\rho>1$ remains unexplored. In this paper, we develop four scheduling algorithms for the four group communications. We derive explicit tight bounds on the latencies of the four communication schedules produced by these algorithms. These latency bounds in general decrease with the number of channels and are also within constant factors of the respective minimum.

\section{Preliminaries}

Let $G=(V, E)$ be an undirected graph. The subgraph of $G$ induced by a subset $U$ of $V$ is denoted by $G[U]$. The maximum (respectively, minimum) degree of $G$ is denoted by $\Delta(G)$ (respectively, $\delta(G)$ ). The inductivity of $G$ is defined by

$$
\delta^{*}(G)=\max _{U \subseteq V} \delta(G[U])
$$

The graph radius of $G$ with respect to a node $v$ is the maximum depth of the breadth-first-search (BFS) tree rooted at $v$. A graph center of $G$ is a node in $G$ with respect to which the graph radius of $G$ is the smallest.

A subset $U$ of $V$ is an independent set of $G$ if no two nodes in $U$ are adjacent. If $U$ is a independent set of $G$ but no proper superset of $U$ is a independent set of $G$, then $U$ is called a maximal independent set (MIS) of $G$. Any node ordering $v_{1}, v_{2}, \cdots, v_{n}$ of $V$ induces an MIS $U$ in the following first-fit manner: Initially, $U=\left\{v_{1}\right\}$. For $i=2$ up to $n$, add $v_{i}$ to $U$ if $v_{i}$ is not adjacent to any node in $U$. A subset $U$ of $V$ is a dominating set of $G$ if each node not in $U$ is adjacent to some node in $U$. Clearly, every MIS of $G$ is also a dominating set of $G$. If $U$ is a dominating set of $G$ and the subgraph of $G$ induced by $U$ is connected, then $U$ is called a connected dominating set (CDS) of $G$.

A (vertex) coloring of $G$ is an assignment of colors to $V$ satisfying that adjacent vertices are assigned with distinct colors. Equivalently, a vertex coloring corresponds to a partition of $V$ into independent sets. First-fit coloring is a simple coloring algorithm. Consider a vertex ordering $\left\langle v_{1}, v_{2}, \cdots, v_{n}\right\rangle$ of $V$. For each $1 \leq i \leq n$, denote

$$
N_{\prec}\left(v_{i}\right)=\left\{v_{j}: 1 \leq j<i, v_{j} \in N\left(v_{i}\right)\right\} .
$$


The first-fit coloring in the ordering $\left\langle v_{1}, v_{2}, \cdots, v_{n}\right\rangle$ use colors represented by natural numbers and runs as follows: Assign the color 1 to $v_{1}$. For $i=2$ up to $n$, assign to $v_{i}$ with the smallest color not used by node in $N_{\prec}\left(v_{i}\right)$. Clearly, the output coloring uses at most $1+\max _{1<i \leq n}\left|N_{\prec}\left(v_{i}\right)\right|$ colors. The parameter $\max _{1<i \leq n}\left|N_{\prec}\left(v_{i}\right)\right|$ is referred to as its inductivity of $\left\langle v_{1}, v_{2}, \cdots, v_{n}\right\rangle$.

Among all vertex orderings, a special vertex ordering, known as smallestlast ordering 13, achieves the smallest inductivity. It is produced iteratively as follows: Initialize $H$ to $G$. For $i=n$ down to 1 , let $v_{i}$ be a vertex of the smallest degree in $H$ and delete $v_{i}$ from $H$. Then the ordering $\left\langle v_{1}, v_{2}, \cdots, v_{n}\right\rangle$ is a smallest-last ordering. Its inductivity is equal to the inductivity of $G$, which is defined by $\delta^{*}(G)=\max _{U \subseteq V} \delta(G[U])$.

Let $D=(V, A)$ be a digraph. The subgraph of $D$ induced by a subset $U$ of $V$ is denoted by $D[U]$. The out-degree (respectively, in-degree) of a node $u$ in $D$ is denoted by $\operatorname{deg}_{D}^{\text {out }}(u)$ (respectively, $\operatorname{deg}_{D}^{i n}(u)$ ). The maximum out-degree (respectively, in-degree) of $D$ is denoted by $\Delta^{\text {out }}(D)$ (respectively, $\Delta^{i n}(D)$ ).

An orientation of an undirected graph $G$ is a digraph obtained from $G$ by imposing an orientation on each edge of $G$. Suppose that the digraph $D=(V, A)$ is an orientation of $G=(V, E)$. We claim that $\delta^{*}(G) \leq 2 \Delta^{\text {out }}(D)$. Indeed, for any $U \subseteq V, D[U]$ contains at least one node $u$ satisfying $\operatorname{deg}_{D[U]}^{i n}(u) \leq$ $\operatorname{deg}_{D[U]}^{\text {out }}(u)$. Thus,

$$
\begin{aligned}
\delta(G[U]) & \leq \operatorname{deg}_{G[U]}(u)=\operatorname{deg}_{D[U]}^{\text {in }}(u)+\operatorname{deg}_{D[U]}^{\text {out }}(u) \\
& \leq 2 \operatorname{deg}_{D[U]}^{\text {out }}(u) \leq 2 \operatorname{deg}_{D}^{\text {out }}(u) \leq 2 \Delta^{\text {out }}(D)
\end{aligned}
$$

So, $\delta^{*}(G) \leq 2 \Delta^{\text {out }}(D)$.

\section{Dominating Tree}

Let $G$ denote the unit-disk graph on $V$, which represents the communication topology. In this section, we describe a rooted spanning tree $T$ of $G$ constructed from the CDS presented in [14. This tree will be used in the routings of all the four group communications. Depending on the type of the group communications, the root of $T$, denoted by $s$, is chosen as follows. For broadcast, $s$ is the source the broadcast; for aggregation or gathering, $s$ is the sink node; for gossiping, $s$ is a graph center of $G$. In either case, we use $L$ to denote the graph radius of $G$ with respect to $s$.

We begin with the construction of a small, short and sparse CDS of $G$ presented in 14. We first select a maximal independent set (MIS) $I$ of $G$ in the first-fit manner in a breadth-first-search (BFS) ordering (with respect to $s$ ) of $V$. All nodes in $I$ form a dominating set, and hence are referred to as dominators. Then, we select a set $C$ of connectors to interconnect $I$ as follows. Let $G^{\prime}$ be the graph on $I$ in which there is edge between two dominators if and only if they have a common neighbor. The radius of $G^{\prime}$ with respect to $s$ is denote by $L^{\prime}$. Clearly, $L^{\prime} \leq L-1$. For each $0 \leq l \leq L^{\prime}$, let $I_{l}$ be the set of dominators of depth $l$ in $G^{\prime}$. Then, $I_{0}=\{s\}$. For each $0 \leq l<L^{\prime}$, let $P_{l}$ be the set of nodes 
adjacent to at least one node in $I_{l}$ and at least one node in $I_{l+1}$, and compute a minimal cover $C_{l} \subseteq P_{l}$ of $I_{l+1}$. Set $C=\bigcup_{l=0}^{L^{\prime}-1} C_{l}$. Then, $I \cup C$ is a CDS of $G$. The following sparse properties of $I \cup C$ were proved in [14]:

$-\left|C_{0}\right| \leq 12$.

- For each $2 \leq l \leq L^{\prime}-1$, each dominator in $I_{l}$ is adjacent to at most 11 connectors in $C_{l}$.

- For each $1 \leq l \leq L^{\prime}-1$, Each connector in $C_{l}$ is adjacent to at most 4 dominators in $I_{l+1}$.

Now, we construct $T$ by specifying the parent of each node other than $s$. First, each dominator in $I_{l}$ with $2 \leq l \leq L^{\prime}$ chooses the neighboring connector of the smallest ID in $C_{l-1}$ as its parent. Second, each connector in $C_{l}$ with $0 \leq l \leq L^{\prime}-1$ chooses the neighboring dominator of the smallest ID in $I_{l}$ as its parent. Third, each other node, referred to as dominatee, chooses the neighboring dominator of the smallest ID as its parent. Clearly, $T$ is a spanning tree, and is called a dominating tree.

We introduce two geometric packing parameters. For any $r>0$, let $\alpha_{r}$ (resp. $\beta_{r}$ ) denote the maximum number of points in a disk (resp., a half-disk) of radius $r$ whose mutual distances are greater than one. Then, by Groemer's Inequality [9],

$$
\begin{aligned}
\alpha_{r} & \leq\left\lfloor\frac{2 \pi}{\sqrt{3}} r^{2}+\pi r\right\rfloor+1, \\
\beta_{r} & \leq\left\lfloor\frac{\pi}{\sqrt{3}} r^{2}+\left(\frac{\pi}{2}+1\right) r\right\rfloor+1 .
\end{aligned}
$$

We use $H_{1}$ to denote the $(\rho+1)$-disk graph on $I$. In other words, a pair of dominators in $I$ are adjacent in $H_{1}$ if and only if their Euclidean distance is at most $\rho+1$. Consider an arbitrary set of dominators $U$. It's easy to show that $\delta\left(H_{1}[U]\right) \leq \beta_{\rho+1}-1$. Therefore, $\delta^{*}\left(H_{1}\right) \leq \beta_{\rho+1}-1$. Consequently for any subset $U$ of dominators, $\delta^{*}\left(H_{1}[U]\right) \leq \beta_{\rho+1}-1$. Hence, the first-fit coloring of $H_{1}[U]$ in the smallest-last ordering uses at most $\beta_{\rho+1}$ colors, and such coloring is simply referred to as the first-fit coloring of $U$.

We also define a conflict graph $\mathrm{H}_{2}$ on $C$ as follows. A pair of connectors $u$ and $v$ are adjacent in $H_{2}$ if and only if either a dominator child of $v$ is within the interference range of $u$, or a dominator child of $u$ is within the interference range of $v$. We claim that $\delta^{*}\left(H_{2}\right) \leq 2\left(\alpha_{\rho}-1\right)$. To prove this claim, we introduce an orientation $\mathrm{D}$ of $\mathrm{H}_{2}$ as follows. Consider an arbitrary edge $u v$ in $H$ and assume that $u$ has smaller ID than $v$. If a dominator child of $v$ is within the interference range of $u$, we orient the edge $u v$ to a link $(u, v)$; otherwise, we orient the edge $u v$ to a link $(v, u)$. It's sufficient to show that $\Delta^{\text {out }}(D) \leq \alpha_{\rho}-1$. Consider a connector $u$ in $D$ with maximum out-degree. Let $X$ denote the set of dominators within the interference range of $u$. Then, $|X| \leq \alpha_{\rho}$. For each out-neighbor $v$ of $u$ in $D, v$ has at least one child in $X$. On the other hand, all child dominators of $u$ are also in $X$. Hence,

$$
\Delta^{\text {out }}(D) \leq|X|-1 \leq \alpha_{\rho}-1 .
$$


So, our claim holds. Therefore, the first-fit coloring of $H_{2}$ in the smallest-last ordering uses at most $2\left(\alpha_{\rho}-1\right)+1=2 \alpha_{\rho}-1$ colors. This coloring is simply referred to as the first-fit coloring of $C$.

Using similar argument, we can show that for $1 \leq l \leq L^{\prime}-1, \delta^{*}\left(H_{2}\left[C_{l}\right]\right) \leq$ $2\left(\alpha_{\rho}-2\right)$. Therefore, the first-fit coloring of $H_{2}\left[C_{l}\right]$ in the smallest-last ordering uses at most $2\left(\alpha_{\rho}-2\right)+1=2 \alpha_{\rho}-3$ colors. This coloring is simply referred to as the first-fit coloring of $C_{l}$.

\section{Broadcast Scheduling}

Let $s$ be the source of the broadcast. We first construct the dominating tree $T$ rooted as $s$ as in Section 3. The routing of the broadcast is the spanning $s$-aborescence oriented from $T$. The broadcast schedule is then partitioned in $2 L^{\prime}+1$ rounds sequentially dedicated to the transmissions by

$$
I_{0}, C_{0}, I_{1}, C_{1}, \cdots, I_{L^{\prime}-1}, C_{L^{\prime}-1}, I_{L^{\prime}}
$$

respectively. The individual rounds are then scheduled as follows:

- In the round for $I_{0}$, only the source node $s$ transmits, and hence this round has only one time-slot.

- In the round for $C_{0}$, we sort $C_{0}$ arbitrarily and let the $i$-th node in $C_{0}$ transmit in the $\lfloor i / \lambda\rfloor$-th time slot at channel $i \bmod \lambda$. Since $\left|C_{0}\right| \leq 12$, this round takes at most $\lceil 12 / \lambda\rceil$ time-slots.

- In the round for $I_{l}$ with $1 \leq l \leq L^{\prime}$, we compute a a first-fit coloring of $I_{l}$, and let each dominator of the $i$-th color transmit in the $\lfloor i / \lambda\rfloor$-th time slot at channel $i \bmod \lambda$. This round takes at most $\left\lceil\beta_{\rho+1} / \lambda\right\rceil$ time-slots.

- In the round for $C_{l}$ with $1 \leq l \leq L^{\prime}-1$, we compute a a first-fit coloring of $C_{l}$, and let each connector of the $i$-th color transmit in the $\lfloor i / \lambda\rfloor$-th time slot at channel $i \bmod \lambda$. This round takes at most $\left\lceil\left(2 \alpha_{\rho}-3\right) / \lambda\right\rceil$ time-slots.

Thus, the latency of the entire broadcast schedule is at most

$$
\begin{aligned}
& 1+\left\lceil\frac{12}{\lambda}\right\rceil+L^{\prime}\left\lceil\frac{\beta_{\rho+1}}{\lambda}\right\rceil+\left(L^{\prime}-1\right)\left\lceil\frac{2 \alpha_{\rho}-3}{\lambda}\right\rceil \\
& \leq\left\lceil\frac{\beta_{\rho+1}}{\lambda}\right\rceil(L-1)+\left\lceil\frac{2 \alpha_{\rho}-3}{\lambda}\right\rceil(L-2)+\left\lceil\frac{12}{\lambda}\right\rceil+1 .
\end{aligned}
$$

Since $\beta_{\rho+1} \geq 12$, it's easy to show that

$$
\left\lceil\frac{\beta_{\rho+1}}{\lambda}\right\rceil(L-1)+\left\lceil\frac{2 \alpha_{\rho}-3}{\lambda}\right\rceil(L-2)+\left\lceil\frac{12}{\lambda}\right\rceil+1 \leq\left(\left\lceil\frac{\beta_{\rho+1}}{\lambda}\right\rceil+\left\lceil\frac{2 \alpha_{\rho}-3}{\lambda}\right\rceil\right) L .
$$

As $L$ is a trivial lower bound on the minimum broadcast latency, the approximation ratio of the our broadcast schedule is at most $\left\lceil\frac{\beta_{\rho+1}}{\lambda}\right\rceil+\left\lceil\frac{2 \alpha_{\rho}-3}{\lambda}\right\rceil$. 


\section{Aggregation Scheduling}

Let $s$ be the sink of the aggregation. Let $\Delta$ denote the maximum degree of $G$, and $L$ be the graph radius of $G$ with respect to $s$. For the trivial case that $L=1$, we simply let all nodes other than $s$ transmit one by one. Such trivial schedule has latency $n-1=\Delta$. Subsequently, we assume that $L>1$. We first construct the dominating tree $T$ rooted as $s$ as in Section 3 . The routing of the aggregation schedule is the spanning inward $s$-aborescence oriented from $T$. Let $W$ denote the set of dominatees. The aggregation schedule is then partitioned in $2 L^{\prime}+1$ rounds sequentially dedicated to the transmissions by

$$
W, I_{L^{\prime}}, C_{L^{\prime}-1}, I_{L^{\prime}-1}, \cdots, C_{1}, I_{1}, C_{0}
$$

respectively. We describe a procedure used by the scheduling in the round for $W$ and the round for each $C_{l}$ with $1 \leq l \leq L^{\prime}-1$.

Let $B$ be a set of links whose receiving endpoints are all dominators. Suppose that $\phi$ is the maximum number of links in $B$ with a common dominator endpoint. We first partition $B$ into $\phi$ subsets $B_{j}$ with $1 \leq j \leq \phi$ such that each dominator is incident to at most one link in each $B_{j}$. The schedule of $B$ is then further partitioned into $\phi$ sub-rounds dedicated to $B_{1}, B_{2},, \cdots, B_{\phi}$ respectively. In the sub-round for $B_{j}$, we compute a first-fit coloring of the dominators incident to the links in $B_{j}$, and then each link in $B_{j}$ whose dominator endpoint receives the $i$-th color is scheduled in the $[i / \lambda\rceil$-th time-slot at the channel $i \bmod \lambda$. Thus, each of the $\phi$ sub-rounds consists of at most $\left\lceil\beta_{\rho+1} / \lambda\right\rceil$ time-slots. Hence, the total number of slots is at most $\phi\left\lceil\beta_{\rho+1} / \lambda\right\rceil$.

Now, we are ready to describe the schedule in the individual rounds.

- In the round for $W$, we adopt the above procedure to produce a schedule in this round. Since each dominator is adjacent to at least one dominatee, the maximum number of nodes in $W$ adjacent to a dominator is at most $\Delta-1$. Hence, this round takes at most $(\Delta-1)\left\lceil\beta_{\rho+1} / \lambda\right\rceil$ time-slots.

- In the round for $C_{l}$ with $1 \leq l \leq L^{\prime}-1$, we also adopt the above procedure to produce a schedule in this round. Since each dominator in $I_{l-1}$ is adjacent to at most 11 connectors in $C_{l}$, this round takes at most $11\left\lceil\beta_{\rho+1} / \lambda\right\rceil$ time-slots.

- In the round for $C_{0}$, all nodes in $C_{0}$ transmit one by one, and thus this round takes at most 12 time-slots.

- In the round for $I_{l}$ with $1 \leq l \leq L^{\prime}$, we compute a a first-fit coloring of $I_{l}$, and let each dominator with the $i$-th color transmit in the $i$-th time slot. This round takes at most $\left\lceil\beta_{\rho+1} / \lambda\right\rceil$ time-slots.

Thus, the latency of the entire aggregation schedule is at most

$$
\begin{aligned}
& (\Delta-1)\left\lceil\frac{\beta_{\rho+1}}{\lambda}\right\rceil+11\left\lceil\frac{\beta_{\rho+1}}{\lambda}\right\rceil\left(L^{\prime}-1\right)+12+L^{\prime}\left\lceil\frac{\beta_{\rho+1}}{\lambda}\right\rceil \\
& =\left\lceil\frac{\beta_{\rho+1}}{\lambda}\right\rceil\left(\Delta+12 L^{\prime}-12\right)+12 \leq\left\lceil\frac{\beta_{\rho+1}}{\lambda}\right\rceil(\Delta+12 L-24)+12 .
\end{aligned}
$$


A trivial lower bound on the minimum aggregation latency is $L$. We claim that $\left\lceil\Delta /\left(\lambda \alpha_{1 /(\rho-1)}\right)\right\rceil$ is also a lower bound for any $\rho>1$. Indeed, let $u$ be a node with maximum degree in $G$, and $S$ be the unit-disk centered at $u$. Then, $S$ contains $\Delta+1$ nodes. If $s$ is not in $S$, then all these $\Delta+1$ nodes in $S$ have to transmit; otherwise, exactly $\Delta$ nodes in $S$ have to transmit. In either case, at least $\Delta$ nodes in $S$ have to transmit. Since all nodes transmitting in the same time-slot at the same channel must be apart from each other by a distance greater than $\rho-1$, at most $\lambda \alpha_{1 /(\rho-1)}$ nodes in $S$ can transmit in a time-slot. Hence, the $\Delta$ transmissions by the nodes in $S$ takes at least $\left\lceil\Delta /\left(\lambda \alpha_{1 /(\rho-1)}\right)\right\rceil$ time-slots. So, our claim holds. Therefore, the approximation bound of the aggregation schedule is at most $\left\lceil\beta_{\rho+1} / \lambda\right\rceil\left(\lambda \alpha_{1 /(\rho-1)}+12\right)$.

\section{$6 \quad$ Gathering Scheduling}

Let $s$ be the sink of the gathering. If $L=1$, then all other nodes transmit to $s$ one by one, and this schedule is optimal. So, we assume subsequently that $L>1$. We first construct the dominating tree of $G$ rooted at $s$. The routing of the gathering schedule is the spanning inward $s$-aborescence oriented from $T$. Our gather schedule utilizes a labelling of the edges of $T$, which is described below.

Let $\left\langle v_{1}, v_{2}, \cdots, v_{n-1}\right\rangle$ be an ordering of $V \backslash\{s\}$ in the descending order of depth in $T$ with ties broken arbitrarily. For $1 \leq i \leq n$, we assign the $j$-th edge in the tree path from $s$ to $v_{j}$ with a label $2(i-1)+j$ (see an example in Figure 1). Clearly, the number of labels received by an edge connecting $v$ and its parent is equal to the number of descendents (including $v$ itself) of $v$ in $T$. If $v$ is connector (respectively, dominator), all labels received by the edge between $v$ and its parent are odd (respectively, even). In addition, all edges across two consecutive layers of the dominating tree receive distinct labels. We further claim that the largest label is $2 n-3$. Consider a node $v_{i}$ and let $h$ be the length of the path from $s$ to $v_{i}$. The maximum label assigned to the edges in the path from $s$ to $v_{i}$ is $2(i-1)+h$. It's sufficient to show that $2(i-1)+h \leq 2 n-3$. Since none of $v_{1}, v_{2}, \cdots, v_{i-1}$ belongs to the path from $s$ to $v_{i}$, we have $h+i-1 \leq n-1$ and hence $i \leq n-h$. Therefore,

$$
2(i-1)+h \leq 2(n-h-1)+h=2 n-h-2 \leq 2 n-3 .
$$

So, the claim holds.

For each $1 \leq k \leq 2 n-3$, let $E_{k}$ denote the set of edges of $T$ which has been assigned with a label $k$, and $A_{k}$ denote the links in the inward $s$-arborescence oriented from the edges in $E_{k}$. Then, for odd (respectively, even) $k$, all the receiving (respectively, transmitting) endpoints of links in $A_{k}$ are dominators. In addition, for each $1 \leq k \leq 2 n-3$, every dominator is incident to at most one link in $A_{k}$.

Now, we are ready to describe the gathering schedule. The schedule are partitioned in $2 n-3$ rounds sequentially dedicated to $A_{2 n-3}, A_{2 n-2}, \cdots, A_{2}, A_{1}$ respectively. For each $1 \leq k \leq 2 n-3$, the round for $A_{k}$ is scheduled as follows. 


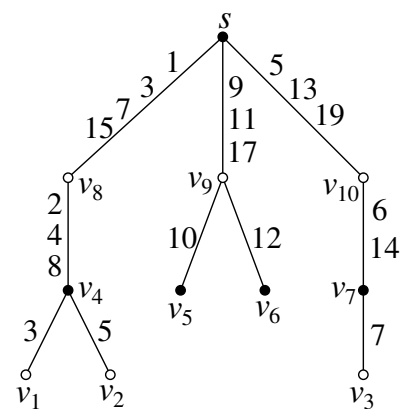

Fig. 1. A multi-labelling of the edges in the dominating tree

We first compute a first-fit distance- $(\rho+1)$ coloring of the dominator endpoints of the links $A_{k}$. Then each link whose dominator endpoint receives the $i$-th color is scheduled in the $[i / \lambda\rceil$-th time-slot at the channel $i \bmod \lambda$.. Thus, each round takes at most $\left\lceil\beta_{\rho+1} / \lambda\right\rceil$ time-slots.

It's obvious that the latency of the gathering schedule is $\left\lceil\beta_{\rho+1} / \lambda\right\rceil(2 n-3)$. Since $n-1$ is a trivial lower bound on the minimum gathering latency, the approximation ratio of the gathering schedule presented in this section is at most $2\left\lceil\beta_{\rho+1} / \lambda\right\rceil$.

\section{Gossiping Scheduling}

Let $s$ be the graph center of $G$. Our gossiping schedule consists of two phases. In the first phase $s$ collects all the packets from all other nodes, and in the second phase $s$ broadcasts all the $n$ packets to all other nodes. We adopt the gathering schedule presented in the previous section for the first phase. In the second phase, the node $s$ disseminates all received packets and its own packet to all other nodes. We present a schedule for the second phase next.

We construct the dominating tree $T$ of $G$ rooted at $s$. The routing of the second phase is the spanning $s$-aborescence oriented from $T$. We compute the first-fit coloring of dominators, and let $k_{1}$ be the number of colors used by this coloring. By proper renumbering of the colors, we assume that $s$ has the first color. We then compute a first-fit coloring of all connectors, and let $k_{2}$ be the number of colors used by this coloring. We group the time-slots into frames of length $\left\lceil k_{1} / \lambda\right\rceil+\left\lceil k_{2} / \lambda\right\rceil$. In each frame, the first $\left\lceil k_{1} / \lambda\right\rceil$ slots form a dominator subframe, and the remaining $\left\lceil k_{2} / \lambda\right\rceil$ slots form a connector subframe. The source node $s$ transmits one packet in each frame. Each connector (respectively, dominator) of color $i$ receiving a packet in a dominator (respectively, connector) subframe transmits the received packet at the channel $i \bmod \lambda$ in the $\lceil i / \lambda\rceil$-th time-slot of the subsequent connector (respectively, dominator) subframe.

The correctness of the above schedule is obvious. The latency of the second phase can be bound as follows. After $n-1$ frames, $s$ transmits the last packet. After another $L^{\prime}$ frames, the last packet reaches all nodes in $I_{L^{\prime}}$. Finally, after 
another dominator sub-frame, the last packet reaches all nodes. So, the total number of time-slots takes by the second phase is at most

$$
\begin{aligned}
& \left(\left\lceil\frac{k_{1}}{\lambda}\right\rceil+\left\lceil\frac{k_{2}}{\lambda}\right\rceil\right)\left(n-1+L^{\prime}\right)+\left\lceil\frac{k_{1}}{\lambda}\right\rceil \leq\left(\left\lceil\frac{k_{1}}{\lambda}\right\rceil+\left\lceil\frac{k_{2}}{\lambda}\right\rceil\right)(n+L-2)+\left\lceil\frac{k_{1}}{\lambda}\right\rceil \\
& \leq\left(\left\lceil\frac{\beta_{\rho+1}}{\lambda}\right\rceil+\left\lceil\frac{2 \alpha_{\rho}-1}{\lambda}\right\rceil\right)(n+L-2)+\left\lceil\frac{\beta_{\rho+1}}{\lambda}\right\rceil .
\end{aligned}
$$

Since the first phase takes at most $\left\lceil\beta_{\rho+1} / \lambda\right\rceil(2 n-3)$ time-slots, the total number of time-slots taken by the two phases is at most

$$
\begin{aligned}
& \left(\left\lceil\frac{\beta_{\rho+1}}{\lambda}\right\rceil+\left\lceil\frac{2 \alpha_{\rho}-1}{\lambda}\right\rceil\right)(n+L-2)+\left\lceil\frac{\beta_{\rho+1}}{\lambda}\right\rceil(2 n-2) \\
& =\left\lceil\frac{\beta_{\rho+1}}{\lambda}\right\rceil(3 n+L-4)+\left\lceil\frac{2 \alpha_{\rho}-1}{\lambda}\right\rceil(n+L-2)
\end{aligned}
$$

Next, we show that the minimum gossiping latency is at least $n-1+L$. The broadcasting of each message requires at least $L$ transmissions. So, the total number of transmissions in any gossiping schedule is at least $n L$. This implies that some node must take at least $L$ transmissions. On the other hand, every node must take $n-1$ receptions. Therefore, some node takes at least $n-1+L$ transmissions and receptions. So, $n-1+L$ is a lower bound on the minimum gossiping latency. Therefore, the approximation ratio of our gossiping schedule is at most $3\left\lceil\beta_{\rho+1} / \lambda\right\rceil+\left\lceil\left(2 \alpha_{\rho}-1\right) / \lambda\right\rceil$.

\section{Summary}

In this paper, we developed four communications schedules for broadcast, aggregation, gathering and gossiping respectively in multi-channel multihop wireless networks under the protocol interference model. Table 1 summarizes the upper bounds on the latencies of these communication schedules. The parameter

\begin{tabular}{|c|c|c|c|c|c|}
\hline Communication & \multicolumn{5}{|c|}{ Upper bound on latency } \\
\hline broadcast & $\frac{\beta_{\rho+1}}{\lambda}$ & $(L-1)$ & \begin{tabular}{l|l}
$+\frac{2 \alpha_{\rho}-3}{\lambda}$ \\
\end{tabular} & $(L-$ & $2)+\left\lceil\frac{12}{\lambda}\right\rceil+1$ \\
\hline aggregation & & $\frac{\beta_{\rho+1}}{\lambda}$ & \multicolumn{3}{|c|}{$(\Delta+12 L-24)+12$} \\
\hline gathering & & & \multicolumn{2}{|c|}{\begin{tabular}{|l|l}
$\frac{\beta_{\rho+1}}{\lambda}$ & $(2 n-3)$ \\
\end{tabular}} & \\
\hline gossiping & $\frac{\beta_{\rho+1}}{\lambda}$ & $(3 n+L$ & $-4)+$ & $\frac{2 \alpha_{\rho}-3}{\lambda}$ & $(n+L-2)$ \\
\hline
\end{tabular}
$L$ is the graph radius with respect to the source of the broadcast, sink of the aggregation, sink of the gathering, and the graph center respectively.

Table 1. Summary on the approximations bounds of the scheduling algorithms 


\section{References}

1. Bermond, J.-C., Galtier, J., Klasing, R., Morales, N., Perennes, S.: Hardness and approximation of gathering in static radio networks. In: Proceedings FAWN 2006 (2006)

2. Bonifaci, V., Korteweg, P., Marchetti-Spaccamela, A., Stougie, L.: An Approximation Algorithm for the Wireless Gathering Problem. In: Arge, L., Freivalds, R. (eds.) SWAT 2006. LNCS, vol. 4059, pp. 328-338. Springer, Heidelberg (2006)

3. Chen, X.J., Hu, X.D., Zhu, J.M.: Minimum data aggregation time problem in wireless sensor networks. In: Jia, X., Wu, J., He, Y. (eds.) MSN 2005. LNCS, vol. 3794, pp. 133-142. Springer, Heidelberg (2005)

4. Chen, Z., Qiao, C., Xu, J., Lee, T.: A Constant Approximation Algorithm for Interference Aware Broadcast in Wireless Networks. In: IEEE INFOCOM 2007 (2007)

5. Dessmark, A., Pelc, A.: Tradeoffs between knowledge and time of communication in geometric radio networks. In: Proceedings of the 13th Annual ACM Symposium on Parallel Algorithms and Architectures (SPAA 2001), Crete, Greece, July 2001, pp. 59-66 (2001)

6. Florens, C., McEliece, R.: Packets distribution algorithms for sensor networks. In: IEEE INFOCOM 2003, pp. 1063-1072 (2003)

7. Gandhi, R., Kim, Y.-A., Lee, S., Ryu, J., Wan, P.-J.: Approximation Algorithms for Data Broadcast in Wireless Networks. In: IEEE INFOCOM Mini-conference 2009 (2009)

8. Gandhi, R., Parthasarathy, S., Mishra, A.: Minimizing broadcast latency and redundancy in ad hoc networks. In: Proceedings of the 4th ACM international symposium on Mobile Ad hoc networking and computing (MobiHoc 2003), pp. 222-232 (2003)

9. Groemer, H.: Über die Einlagerung von Kreisen in einen konvexen Bereich. Math. Z. 73, 285-294 (1960)

10. Huang, S.C.-H., Du, H., Park, E.-K.: Minimum-latency gossiping in multi-hop wireless networks. In: ACM Mobihoc 2008 (2008)

11. Huang, C.-H., Wan, P.-J., Deng, J., Han, Y.S.: Broadcast Scheduling in Interference Environment. IEEE Transactions on Mobile Computing 7(11), 1338-1348 (2008)

12. Huang, S.C.-H., Wan, P.-J., Vu, C.T., Li, Y., Yao, F.: Nearly Constant Approximation for Data Aggregation Scheduling in Wireless Sensor Networks. In: IEEE INFOCOM 2007 (2007)

13. Matula, D.W., Beck, L.L.: Smallest-last ordering and clustering and graph coloring algorithms. Journal of the Association of Computing Machinery 30(3), 417-427 (1983)

14. Wan, P.-J., Huang, C.-H., Wang, L., Wan, Z.-Y., Jia, X.: Minimum-Latency Aggregation Scheduling in Multihop Wireless Networks. In: ACM MOBIHOC 2009 (2009) 